\title{
Sensitivity of D and B Meson Decays to their Transition Form Factors
}

\author{
Dae Sung Hwang* \\ Department of Physics, Sejong University, Seoul 143-747, Korea \\ E-mail: 'dshwang@kunja. sejong.ac.kn
}

Abstract: We study the sensitivity of the $d \Gamma / d q^{2}$ spectra and the branching fractions of the $D$ meson exclusive semileptonic decays to form factor models. We also analyze the ratio of the branching fractions $\mathcal{B}\left(D^{0} \rightarrow K^{-}\right.$(or $\left.\left.\pi^{-}\right) \pi^{+}\right)$and $\mathcal{B}\left(D^{0} \rightarrow K^{-}\left(\right.\right.$or $\left.\left.\pi^{-}\right) e^{+} \nu\right)$ using the factorization assumption and find that it is implied that $F_{1}\left(q^{2}\right)$ is of dipole type instead of simple pole type which is usually assumed in experimental analyses. If we emply dipole type $F_{1}\left(q^{2}\right)$, the experimentally extracted value of $F_{1}^{D K}(0)$ becomes 0.64 , whereas 0.75 was obtained by assuming monopole type $F_{1}\left(q^{2}\right)$. We study $\bar{B}^{0} \rightarrow D^{(*)+} l^{-} \bar{\nu}$ using experimentally measured form factors, and find the $d \Gamma / d q^{2}$ spectra and branching fractions for $l=e, \mu$ and $\tau$.

\section{Introduction}

The mechanism of CP-violation through the complex phase of the CKM three family mixing matrix $\left[\begin{array}{l}1 \\ 1\end{array}\right]$ is presently considered standard for the $\mathrm{CP}$-violation. In order to measure the CKM matrix elements accurately, it is important to know the hadronic form factors of the transition matrix elements reliably. For the heavy to heavy transitions the heavy quark effective theory provides good information for the form factors. However, for the heavy to light transitions the understanding of the form factors is still limited; this fact hinders the extractions of the CKM matrix elements from experimental results significantly. At the same time, we will be able to have important clues for the internal structures of hadrons by knowing these form factors well. Semileptonic decay processes are good sources for the knowledge of the form factors both experimentally and theoretically. The lepton mass effects in heavy meson exclusive semileptonic decays were studied by Körner and Schuler [iñin

We derive the formulas for $d \Gamma / d q^{2}$ with nonzero lepton mass in forms which are efficient to

*Do-Won Kim, Department of Physics, Kangnung National University, Kangnung 210-702, Korea study the form factor dependences. This formula for the pseudoscalar to pseudoscalar transition was also given by Khodjamirian et al. By using these formulas we study the $d \Gamma / d q^{2}$ spectra and branching fractions of the exclusive semileptonic $D$ meson decays: $D^{0} \rightarrow K^{(*)-} e^{+} \nu$, $D^{0} \rightarrow K^{(*)-} \mu^{+} \nu, D^{0} \rightarrow \pi^{-}\left(\right.$or $\left.\rho^{-}\right) e^{+} \nu$ and $D^{0} \rightarrow \pi^{-}\left(\right.$or $\left.\rho^{-}\right) \mu^{+} \nu$. In this anslysis we employ three models of form factors and show how the results are influenced by the difference of form factors. For the decays to a pseudoscalar meson and a lepton pair, the results are sensitive to the property of the form factor $F_{1}\left(q^{2}\right)$. For the decays to a vector meson and a lepton pair, the results are sensitive to the form factor $A_{1}\left(q^{2}\right)$, and not to the other ones $\left(A_{0}\left(q^{2}\right), A_{2}\left(q^{2}\right)\right.$ and $\left.V\left(q^{2}\right)\right)$. We also analyze the experimental results of the branching fractions $\mathcal{B}\left(D^{0} \rightarrow K^{-}\left(\right.\right.$or $\left.\left.\pi^{-}\right) \pi^{+}\right)$and $\mathcal{B}\left(D^{0} \rightarrow K^{-}\right.$(or $\left.\left.\pi^{-}\right) e^{+} \nu\right)$, and show that it is implied that the form factor $F_{1}\left(q^{2}\right)$ of the $D$ to $K$ and the $D$ to $\pi$ transitions are of dipole type, instead of simple pole type which is commonly assumed in the studies of the $D$ meson decays.

\section{Semileptonic Decays}

From Lorentz invariance one finds the decompo- 
sition of the hadronic matrix element for pseudoscalar to pseudoscalar meson transition in terms of hadronic form factors:

$$
\begin{aligned}
& <P(p)\left|J_{\mu}\right| P(P)> \\
= & \left((P+p)_{\mu}-\frac{M^{2}-m^{2}}{q^{2}} q_{\mu}\right) F_{1}\left(q^{2}\right) \\
& +\frac{M^{2}-m^{2}}{q^{2}} q_{\mu} F_{0}\left(q^{2}\right),
\end{aligned}
$$

where $J_{\mu}=\bar{q}^{\prime} \gamma_{\mu}\left(1-\gamma_{5}\right) q$. We use the following notations: $M$ represents initial meson mass, $m$ final meson mass, $m_{l}$ lepton mass, $P$ initial meson momentum, $p$ final meson momentum, and $q_{\mu}=(P-p)_{\mu}$. The form factors $F_{1}\left(q^{2}\right)$ and $F_{0}\left(q^{2}\right)$ correspond to $1^{-}$and $0^{+}$exchanges, respectively. At $q^{2}=0$ we have the constraint $F_{1}(0)=F_{0}(0)$, since the hadronic matrix element in (2.1) is nonsingular at this kinematic point.

The $q^{2}$ distribution of the semileptonic decay $D^{0} \rightarrow K^{-} l^{+} \nu$ is given in terms of the hadronic form factors $F_{1}\left(q^{2}\right)$ and $F_{0}\left(q^{2}\right)$ as:

$$
\begin{aligned}
& \frac{d \Gamma\left(D^{0} \rightarrow K^{-} l^{+} \nu\right)}{d q^{2}}=\frac{G_{F}^{2}}{24 \pi^{3}}\left|V_{c s}\right|^{2} \times \\
& K\left(q^{2}\right)\left(1-\frac{m_{l}^{2}}{q^{2}}\right)^{2}\left[\left(K\left(q^{2}\right)\right)^{2}\left(1+\frac{1}{2} \frac{m_{l}^{2}}{q^{2}}\right)\left|F_{1}\left(q^{2}\right)\right|^{2}\right. \\
& \left.+M^{2}\left(1-\frac{m^{2}}{M^{2}}\right)^{2} \frac{3}{8} \frac{m_{l}^{2}}{q^{2}}\left|F_{0}\left(q^{2}\right)\right|^{2}\right],
\end{aligned}
$$

where $K\left(q^{2}\right)$, momentum of the final meson in the $D$ meson rest frame, is given by

$$
K\left(q^{2}\right)=\frac{1}{2 M}\left(\left(M^{2}+m^{2}-q^{2}\right)^{2}-4 M^{2} m^{2}\right)^{\frac{1}{2}},
$$

and the physically allowed range of $q^{2}$ is given by

$$
m_{l}^{2} \leq q^{2} \leq(M-m)^{2} .
$$

From Lorentz invariance one finds the decomposition of the hadronic matrix element for pseudoscalar to vector meson transition in terms of hadronic form factors:

$$
\begin{aligned}
& <V(p)\left|J_{\mu}\right| P(P)> \\
= & \varepsilon^{* \nu}(p)\left((M+m) g_{\mu \nu} A_{1}\left(q^{2}\right)-2 \frac{P_{\mu} P_{\nu}}{M+m} A_{2}\left(q^{2}\right)\right. \\
& \left.+\frac{q_{\mu} P_{\nu}}{M+m} A_{3}\left(q^{2}\right)+i \varepsilon_{\mu \nu \rho \sigma} \frac{P^{\rho} p^{\sigma}}{M+m} V\left(q^{2}\right)\right),
\end{aligned}
$$

where $\varepsilon_{0123}=1$ and

$$
\begin{aligned}
& 2 m A_{0}\left(q^{2}\right)=(M+m) A_{1}\left(q^{2}\right) \\
& -\frac{M^{2}-m^{2}+q^{2}}{M+m} A_{2}\left(q^{2}\right)+\frac{q^{2}}{M+m} A_{3}\left(q^{2}\right) .
\end{aligned}
$$

The form factors $V\left(q^{2}\right), A_{1}\left(q^{2}\right), A_{2}\left(q^{2}\right)$ and $A_{0}\left(q^{2}\right)$ correspond to $1^{-}, 1^{+}, 1^{+}$and $0^{-}$exchanges, respectively. At $q^{2}=0$ we have the constraint $2 m A_{0}(0)=(M+m) A_{1}(0)-(M-m) A_{2}(0)$, since the hadronic matrix element in (2.5) is nonsingular at this kinematic point.

After some calculations [i] tion of the semileptonic decay $D^{0} \rightarrow K^{*-} l^{+} \nu$ is given in terms of the hadronic form factors $A_{1}\left(q^{2}\right), A_{2}\left(q^{2}\right), A_{3}\left(q^{2}\right)$ and $V\left(q^{2}\right)$ as:

$$
\begin{aligned}
& \frac{d \Gamma\left(D^{0} \rightarrow K^{*-} l^{+} \nu\right)}{d q^{2}} \\
& =\frac{G_{F}^{2}}{32 \pi^{3}}\left|V_{c s}\right|^{2} \frac{1}{M^{2}} K\left(q^{2}\right)\left(1-\frac{m_{l}^{2}}{q^{2}}\right)^{2} \times \\
& \left\{| A _ { 1 } ( q ^ { 2 } ) | ^ { 2 } \frac { ( M + m ) ^ { 2 } } { m ^ { 2 } } \left[\frac{1}{3}(M K)^{2}\left(1-\frac{m_{l}^{2}}{q^{2}}\right)\right.\right. \\
& \left.+q^{2} m^{2}+(M K)^{2} \frac{m_{l}^{2}}{q^{2}}+\frac{1}{2} m^{2} m_{l}^{2}\right] \\
& +\operatorname{Re}\left(A_{1}\left(q^{2}\right) A_{2}^{*}\left(q^{2}\right)\right)\left[-\frac{M^{2}-m^{2}-q^{2}}{m^{2}}\right. \\
& {\left[\frac{2}{3}(M K)^{2}\left(1-\frac{m_{l}^{2}}{q^{2}}\right)+2(M K)^{2} \frac{m_{l}^{2}}{q^{2}}\right.} \\
& \left.\left.+\frac{1}{2}\left(M^{2}+m^{2}-q^{2}\right) m_{l}^{2}\right]+\left(M^{2}-m^{2}+q^{2}\right) m_{l}^{2}\right] \\
& +\left|A_{2}\left(q^{2}\right)\right|^{2} \frac{1}{(M+m)^{2} m^{2}}(M K)^{2} \\
& {\left[\frac{4}{3}(M K)^{2}\left(1-\frac{m_{l}^{2}}{q^{2}}\right)+4(M K)^{2} \frac{m_{l}^{2}}{q^{2}}+2 M^{2} m_{l}^{2}\right]} \\
& +\left|V\left(q^{2}\right)\right|^{2} \frac{q^{2}}{(M+m)^{2}}\left[\frac{8}{3}(M K)^{2}\left(1-\frac{m_{l}^{2}}{q^{2}}\right)\right. \\
& \left.+4(M K)^{2} \frac{m_{l}^{2}}{q^{2}}\right] \\
& +\left|A_{3}\left(q^{2}\right)\right|^{2} \frac{q^{2}}{(M+m)^{2} m^{2}} \frac{1}{2}(M K)^{2} m_{l}^{2} \\
& -\operatorname{Re}\left(A_{3}\left(q^{2}\right) A_{2}^{*}\left(q^{2}\right)\right) \frac{1}{(M+m)^{2} m^{2}} \\
& \left(M^{2}-m^{2}+q^{2}\right)(M K)^{2} m_{l}^{2} \\
& \left.+\operatorname{Re}\left(A_{3}\left(q^{2}\right) A_{1}^{*}\left(q^{2}\right)\right) \frac{1}{m^{2}}(M K)^{2} m_{l}^{2}\right\} \text {. }
\end{aligned}
$$

When we take $m_{l} \rightarrow 0$ in (2.7i), it agrees with the

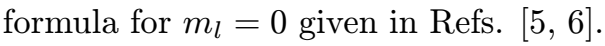

In the case of the $B$ to $D$ meson (heavy to heavy) transition, the heavy quark effective theory (HQET) gives the useful relations between the relevant form factors $[\overline{[} \bar{i}]]$ :

$$
F_{1}\left(q^{2}\right)=V\left(q^{2}\right)=A_{0}\left(q^{2}\right)=A_{2}\left(q^{2}\right)
$$


Table 1: The values of the power of pole for the three models used in this paper.

\begin{tabular}{|c|c|c|c|c|c|c|}
\hline & $n_{F_{1}}$ & $n_{F_{0}}$ & $n_{V}$ & $n_{A_{1}}$ & $n_{A_{2}}$ & $n_{A_{0}}$ \\
\hline WSB & 1 & 1 & 1 & 1 & 1 & 1 \\
Model I & 1 & 0 & 1 & 0 & 1 & 1 \\
Model II & 2 & 1 & 2 & 1 & 2 & 2 \\
\hline
\end{tabular}

Table 2: The values of pole masses $(\mathrm{GeV})$ used in numerical calculations.

\begin{tabular}{|c|c|c|c|c|}
\hline Current & $m\left(0^{-}\right)$ & $m\left(1^{-}\right)$ & $m\left(0^{+}\right)$ & $m\left(1^{+}\right)$ \\
& $A_{0}$ & $F_{1} V$ & $F_{0}$ & $A_{1} A_{2}$ \\
\hline $\bar{s} c$ & 1.97 & 2.11 & 2.60 & 2.53 \\
$\bar{d} c$ & 1.87 & 2.01 & 2.47 & 2.42 \\
\hline
\end{tabular}

Table 3: The values of the form factors at $q^{2}=0$ used in numerical calculations.

\begin{tabular}{|c|c|c|c|c|c|}
\hline & $F_{1}(0)$ & $V(0)$ & $A_{1}(0)$ & $A_{2}(0)$ & $A_{0}(0)$ \\
\hline $\bar{s} c$ & 0.762 & 1.226 & 0.880 & 1.147 & 0.733 \\
$\bar{d} c$ & 0.692 & 1.225 & 0.775 & 0.923 & 0.669 \\
\hline
\end{tabular}

$$
\begin{aligned}
& =\frac{M+m}{2 \sqrt{M m}} \mathcal{F}(y), \\
F_{0}\left(q^{2}\right) & =A_{1}\left(q^{2}\right)=\frac{2 \sqrt{M m}}{M+m} \frac{y+1}{2} \mathcal{F}(y),
\end{aligned}
$$

where $y=\left(M^{2}+m^{2}-q^{2}\right) /(2 M m)=E_{D^{(*)}} / m$ $\left(E_{D^{(*)}}\right.$ is the energy of $D^{(*)}$ meson in the $B$ meson rest frame), and $\mathcal{F}(y)$ is a form factor which becomes the Isgur-Wise function in the infinite heavy quark mass limit. When we use the relations $(2.81)$, for $m_{l}=0$ the formula $(2.7)$ becomes the well-known formula for the $B$ to $D^{*}$ transition:

$$
\begin{aligned}
& \frac{d \Gamma\left(\bar{B}^{0} \rightarrow D^{*+} l^{-} \bar{\nu}\right)}{d q^{2}} \\
= & \frac{G_{F}^{2}}{48 \pi^{3}}\left|V_{c b}\right|^{2} m^{3}(M-m)^{2} \sqrt{y^{2}-1}(y+1)^{2} \\
& \times\left\{1+\frac{4 y}{y+1} \frac{1-2 y r+r^{2}}{(1-r)^{2}}\right\}\left(\mathcal{F}_{D^{*}}(y)\right)^{2}
\end{aligned}
$$

where $r=m / M$.

\section{3. $D^{0} \rightarrow K^{(*)-} l^{+} \nu$}

For the form factors concerned with the exclusive semileptonic decays of $D$ meson, we can not use the relations $(2 . \overline{8})$ of the HQET. Therefore, in the study of $D$ meson decays we use models for form factors. The pole-dominance idea suggests the following $q^{2}$ dependence of the form factors [8]1]:

$$
f_{i}\left(q^{2}\right)=f_{i}(0) \frac{1}{\left(1-\frac{q^{2}}{m_{f_{i}}^{2}}\right)^{n_{f_{i}}}},
$$

where $n_{f_{i}}$ and $m_{f_{i}}$ are corresponding power and pole mass of the form factors $f_{i}\left(q^{2}\right)$, respectively. The WSB model [i]; adopts $n_{f_{i}}=1$. However, the exact values of $n_{f_{i}}$ are not known. The relations (2.8) of the HQET gives the following approximate relation among the powers of the form factors for the heavy to heavy transitions:

$$
n_{F_{1}}=n_{V}=n_{A_{0}}=n_{A_{2}}=n_{F_{0}}+1=n_{A_{1}}+1 .
$$

Non-perturbative analysis of QCD [9 $[\overline{9}]$ suggests the same relation as ( $3 . \overline{2})$ for the form factors of the heavy to light transitions. The lattice calculations also show that the form factors $F_{1}$, $V$ and $A_{0}$ are more rapidly increasing functions

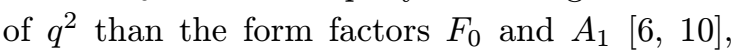
which favors the relation $(\underline{3} \cdot \overline{2})$. Therefore, we will study two other models incorporating the relation (13.2), as well as the WBS model, for the study of the exclusive semileptonic decays of $D$ meson. Table 1 shows the values of the powers $n_{f_{i}}$ of the three models which we use in this work. For the values of the pole masses and those of the form factors at $q^{2}=0$, we use the values shown in Table 2 and 3, which were given by Wirbel, Stech and Bauer [isi]. Their precise values are not known and they should be different for each of the three models. However, their exact values are not crucial in our present work since the purpose of this paper is to clarify how the $d \Gamma / d q^{2}$ spectra and the branching fractions depend on the form factors employed, and to show how experimental extractions of the physical quantities from given data are influenced.

For $D^{0} \rightarrow K^{-} l^{+} \nu$, we use the formula (2.2) in our calculation. The $d \Gamma\left(D^{0} \rightarrow K^{-} l^{+} \nu\right) / d q^{2}$ spectrum and branching fractions are presented in Figure 1 and Table 4, for each of the three 
models: WSB, Model I and Model II explained in Table 1. We find that the shape of spectrum of Model II is different from those of WSB and Model I in Figure 1. That is, the value of $n_{F_{1}}$ determines the shape of spectrum. The experimental result of the E687 Collaboration [1] in for this spectrum shows that the experimental situation is marginal at present; it will be enough to clarify which curve in Figure 1 agrees with the experiment if the experimental error bars are reduced by factor of 3 or 4 . The result of FOCUS/E831 is expected to come out within maybe a year from about 30 times the statistics of E687 [121]. Then we will be able to tell which model of the form factors agrees with the experiment in the near future.

In the experimental extraction of the value of $F_{1}^{D K}(0)$, the simple pole of the form factors

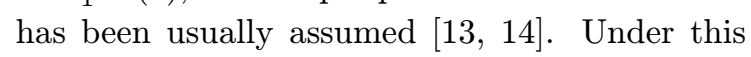
assumption $F_{1}^{D K}(0)=0.75 \pm 0.02 \pm 0.02$ was extracted [1 13 "] from the experimentally measured branching fraction $\mathcal{B}\left(D^{0} \rightarrow K^{-} e^{+} \nu\right)=(3.68 \pm$ $0.21) \times 10^{-2}$. (In Ref. [1 $144_{1}^{\prime \prime}, F_{1}^{D K}(0)=0.76 \pm 0.03$ was presented.) However, if we assume Model II which has the dipole form factor for $F_{1}\left(q^{2}\right)$, we would get

$$
\begin{aligned}
F_{1}^{D K}(0) & =0.75 \times \sqrt{\frac{3.49 \times 10^{-2}}{4.78 \times 10^{-2}}} \\
& =0.75 \times 0.85=0.64
\end{aligned}
$$

for the mean value, with $F_{1}^{D K}(0)=0.64 \pm 0.03$. In $(\overline{3} . \overline{3})$ the ratio inside the square root comes from the results in Table 4 of the branching fraction $\mathcal{B}\left(D^{0} \rightarrow K^{-} e^{+} \nu\right)$ for WSB $\left(n_{F_{1}}=1\right)$ and Model II $\left(n_{F_{1}}=2\right)$, which were obtained by using the same value of $F_{1}^{D K}(0)$. This ratio is equiva-

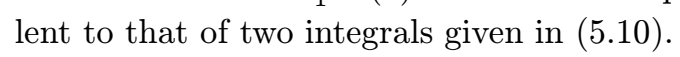

For $D^{0} \rightarrow K^{*-} l^{+} \nu$, we use the formula (2.7) with non-zero lepton mass. The obtained spectra and branching fractions are presented in Table 5 . We find that the results of WSB and Model II are almost the same, and they are significantly different from the results of Model I. This fact implies that the value of $n_{A_{1}}$ is the significant quantity that determines the spectra and branching fractions of $D^{0} \rightarrow K^{*-} l^{+} \nu$.

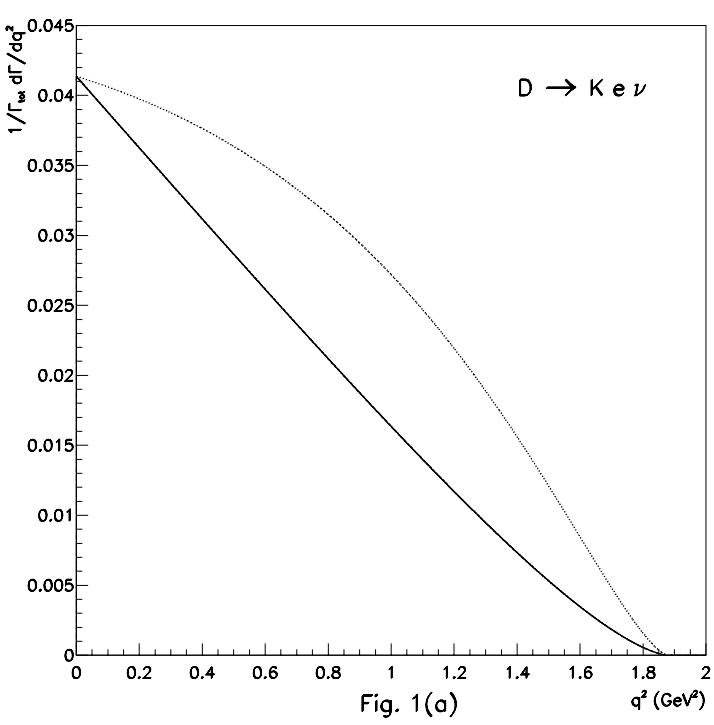

Figure 1: $i\left(1 / \Gamma_{t o t}\right)\left(d \Gamma / d q^{2}\right)$ of $D^{0} \rightarrow K^{-} e^{+} \nu$ for three models: solid line for WSB, dashed for Model I (solid and dashed lines overlap in this figure), and dotted for Model II.

Table 4: The obtained branching fractions and their ratios for $D^{0} \rightarrow K^{-} l^{+} \nu$.

\begin{tabular}{|c|c|c|}
\hline & $\mathcal{B}\left(D^{0} \rightarrow K^{-} e^{+} \nu\right)$ & $\mathcal{B}\left(D^{0} \rightarrow K^{-} \mu^{+} \nu\right)$ \\
\hline WSB & $3.49 \times 10^{-2}$ & $3.41 \times 10^{-2}$ \\
I & $3.49 \times 10^{-2}$ & $3.38 \times 10^{-2}$ \\
II & $4.78 \times 10^{-2}$ & $4.67 \times 10^{-2}$ \\
\hline
\end{tabular}

Table 5: The obtained branching fractions and their ratios for $D^{0} \rightarrow K^{*-} l^{+} \nu$.

\begin{tabular}{|c|c|c|}
\hline & $\mathcal{B}\left(D^{0} \rightarrow K^{*-} e^{+} \nu\right)$ & $\mathcal{B}\left(D^{0} \rightarrow K^{*-} \mu^{+} \nu\right)$ \\
\hline WSB & $3.88 \times 10^{-2}$ & $3.68 \times 10^{-2}$ \\
I & $3.28 \times 10^{-2}$ & $3.10 \times 10^{-2}$ \\
II & $3.85 \times 10^{-2}$ & $3.66 \times 10^{-2}$ \\
\hline
\end{tabular}

4. $D^{0} \rightarrow \pi^{-}\left(\right.$or $\left.\rho^{-}\right) l^{+} \nu$

For $D^{0} \rightarrow \pi^{-} l^{+} \nu$, we use the formula (2.2.20) with the replacement of $V_{c s}$ by $V_{c d}$. The obtained spectra and branching fractions are presented in Figure 2 and Table 6 . We find that the branching fractions of Model II are about twice those 
of WSB and Model I. Therefore, if we determine the value of $F_{1}^{D \pi}(0)$ from an experimentally measured branching fraction of $D^{0} \rightarrow \pi^{-} l^{+} \nu$, the value of $F_{1}^{D \pi}(0)$ determined with Model II will be about $1 / \sqrt{2}$ times its value determined with WSB or Model I. From Table 4 and 6 , we also find that the ratio $\mathcal{B}\left(D^{0} \rightarrow \pi^{-} l^{+} \nu\right) / \mathcal{B}\left(D^{0} \rightarrow K^{-} l^{+} \nu\right)$ from Model II is significantly bigger than that from WSB or Model I, but its present experimental result $0.101 \pm 0.020 \pm 0.003[1 \overline{1} \overline{1}]$ can not discriminate them yet.

For $D^{0} \rightarrow \rho^{-} l^{+} \nu$, we use the formula (2.7\%) with the replacement of $V_{c s}$ by $V_{c d}$. The obtained spectra and branching fractions are presented in Table 7. We find that the results of WSB and Model II are almost the same, and they are much different from the results of Model I. Therefore, like the $D^{0} \rightarrow K^{*-} l^{+} \nu$ case, the property of the form factor $A_{1}\left(q^{2}\right)$ determines the spectra and branching fractions, and the results are not sensitive to the other form factors $\left(A_{0}\left(q^{2}\right), A_{2}\left(q^{2}\right)\right.$ and $\left.V\left(q^{2}\right)\right)$.

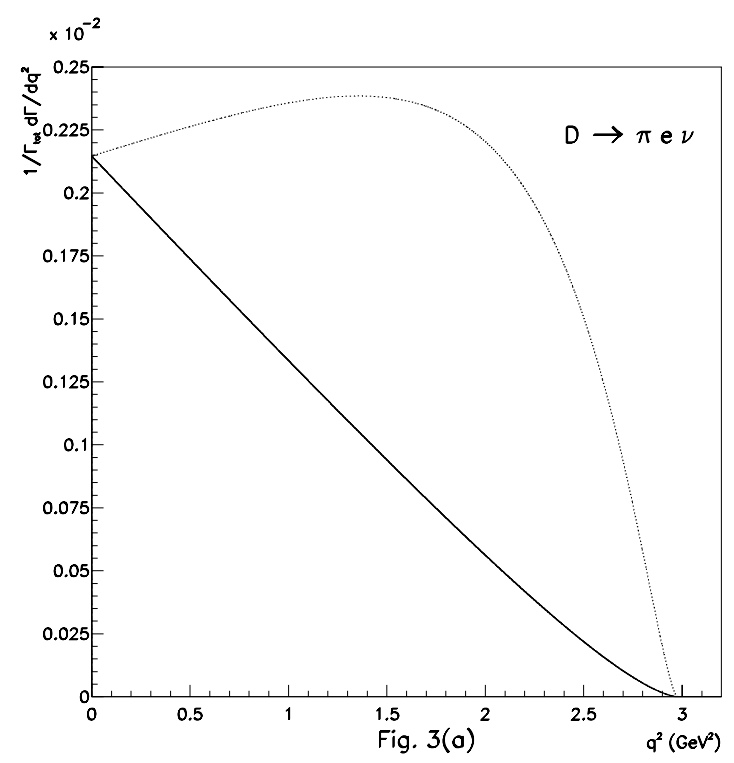

Figure 2: $\left(1 / \Gamma_{t o t}\right)\left(d \Gamma / d q^{2}\right)$ of $D^{0} \rightarrow \pi^{-} e^{+} \nu$ for three models: solid line for WSB, dashed for Model $I$ (solid and dashed lines overlap in this figure), and dotted for Model II.
Table 6: The obtained branching fractions and their ratios for $D^{0} \rightarrow \pi^{-} l^{+} \nu$

\begin{tabular}{|c|c|c|}
\hline & $\mathcal{B}\left(D^{0} \rightarrow \pi e^{+} \nu\right)$ & $\mathcal{B}\left(D^{0} \rightarrow \pi \mu^{+} \nu\right)$ \\
\hline WSB & $2.92 \times 10^{-3}$ & $2.88 \times 10^{-3}$ \\
I & $2.92 \times 10^{-3}$ & $2.86 \times 10^{-3}$ \\
II & $5.94 \times 10^{-3}$ & $5.86 \times 10^{-3}$ \\
\hline
\end{tabular}

Table 7: The obtained branching fractions and their ratios for $D^{0} \rightarrow \rho^{-} l^{+} \nu$.

\begin{tabular}{|c|c|c|}
\hline & $\mathcal{B}\left(D^{0} \rightarrow \rho e^{+} \nu\right)$ & $\mathcal{B}\left(D^{0} \rightarrow \rho \mu^{+} \nu\right)$ \\
\hline WSB & $2.77 \times 10^{-3}$ & $2.66 \times 10^{-3}$ \\
I & $2.19 \times 10^{-3}$ & $2.10 \times 10^{-3}$ \\
II & $2.77 \times 10^{-3}$ & $2.66 \times 10^{-3}$ \\
\hline
\end{tabular}

\section{Implications of Experimental Results}

In this section we compare the exclusive semileptonic decays and the two-body hadronic decays using the factorization assumption. We start by recalling the relevant effective weak Hamiltonian for the two-body hadronic decay $D^{0} \rightarrow K^{-} \pi^{+}$:

$$
\begin{gathered}
\mathcal{H}_{\mathrm{eff}}=\frac{G_{F}}{\sqrt{2}} V_{c s}^{*} V_{u d}\left[C_{1}(\mu) \mathcal{O}_{1}+C_{2}(\mu) \mathcal{O}_{2}\right]+\text { H.C. } \\
\mathcal{O}_{1}=\left(\bar{u} \Gamma^{\rho} d\right)\left(\bar{s} \Gamma_{\rho} c\right), \quad \mathcal{O}_{2}=\left(\bar{s} \Gamma^{\rho} d\right)\left(\bar{u} \Gamma_{\rho} c\right),
\end{gathered}
$$

where $G_{F}$ is the Fermi coupling constant, $V_{c s}$ and $V_{u d}$ are corresponding Cabibbo-KobayashiMaskawa (CKM) matrix elements and $\Gamma_{\rho}=\gamma_{\rho}(1-$ $\left.\gamma_{5}\right)$. The Wilson coefficients $C_{1}(\mu)$ and $C_{2}(\mu)$ incorporate the short-distance effects arising from the renormalization of $\mathcal{H}_{\text {eff }}$ from $\mu=m_{W}$ to $\mu=O\left(m_{c}\right)$. In the factorization assumption,

$$
\begin{aligned}
\mathcal{H}_{\mathrm{eff}}= & \frac{G_{F}}{\sqrt{2}} V_{c s}^{*} V_{u d}\left(a_{1}\left[\bar{u} \Gamma^{\rho} d\right]_{H}\left[\bar{s} \Gamma_{\rho} c\right]_{H}\right. \\
& \left.+a_{2}\left[\bar{s} \Gamma^{\rho} d\right]_{H}\left[\bar{u} \Gamma_{\rho} c\right]_{H}\right)+ \text { H.C. },
\end{aligned}
$$

where the subscript $H$ stands for hadronic implying that the Dirac bilinears inside the brackets be treated as interpolating fields for the mesons. The phenomenological parameters $a_{1}$ and $a_{2}$ are related to $C_{1}$ and $C_{2}$ by $a_{1}=C_{1}+\frac{1}{N_{c}} C_{2}$ and $a_{2}=C_{2}+\frac{1}{N_{c}} C_{1}$ [י] 
and $a_{2}$ for $D$ meson decays are given by [1] $\left.{ }^{-1}{ }_{1}\right]$

$$
a_{1}=1.10 \pm 0.05, \quad a_{2}=-0.49 \pm 0.04 .
$$

By using (2.11), (5.5i) and $\left.<0\left|\Gamma_{\mu}\right| \pi^{-}(q)\right\rangle=$ $i q_{\mu} f_{\pi^{-}}$, we get the following formula for the branching ratio of the process $D^{0} \rightarrow K^{-} \pi^{+}$:

$$
\begin{aligned}
& \mathcal{B}\left(D^{0} \rightarrow K^{-} \pi^{+}\right)=\left(\frac{G_{F} m_{D}^{2}}{\sqrt{2}}\right)^{2}\left|V_{u d}\right|^{2} \\
& \times \frac{1}{8 \pi} \frac{m_{D}}{\Gamma_{D}} a_{1}^{2} \frac{f_{\pi}^{2}}{m_{D}^{2}}\left|V_{c s} F_{0}^{D K}\left(m_{\pi}^{2}\right)\right|^{2}\left(1-\frac{m_{K}^{2}}{m_{D}^{2}}\right)^{2} \\
& \times \frac{1}{2}\left[\left(1-\left(\frac{m_{K}+m_{\pi}}{m_{D}}\right)^{2}\right)\left(1-\left(\frac{m_{K}-m_{\pi}}{m_{D}}\right)^{2}\right)\right]^{\frac{1}{2}} .
\end{aligned}
$$

On the other hand, from $(2.2)$ and (3.1) the branching ratio $\mathcal{B}\left(B^{0} \rightarrow K^{-} e^{+} \nu\right)$ is given by

$$
\begin{aligned}
& \mathcal{B}\left(D^{0} \rightarrow K^{-} e^{+} \nu\right) \\
= & \left(\frac{G_{F} m_{D}^{2}}{\sqrt{2}}\right)^{2} \frac{m_{D}}{\Gamma_{D}} \frac{2}{192 \pi^{3}}\left|V_{c s} F_{1}^{D K}(0)\right|^{2} \times I^{D K},
\end{aligned}
$$

where the dimensionless integral $I^{D K}$ is given by

$$
I^{D K}=\int_{0}^{\left(1-\frac{m_{K}}{m_{D}}\right)^{2}} d x \frac{\left(\left(1+\frac{m_{K}^{2}}{m_{D}^{2}}-x\right)^{2}-4 \frac{m_{K}^{2}}{m_{D}^{2}}\right)^{\frac{3}{2}}}{\left(1-\frac{m_{D}^{2}}{m_{F_{1}}} x\right)^{2 n_{F_{1}}}} .
$$

In the above, we neglected the electron mass.

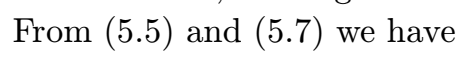

$$
\begin{aligned}
& \frac{\mathcal{B}\left(D^{0} \rightarrow K^{-} \pi^{+}\right)}{\mathcal{B}\left(D^{0} \rightarrow K^{-} e^{+} \nu\right)}=6 \pi^{2}\left|V_{u d}\right|^{2} \frac{f_{\pi}^{2}}{m_{D}^{2}}\left(1-\frac{m_{K}^{2}}{m_{D}^{2}}\right)^{2} \\
& \times\left[\left(1-\left(\frac{m_{K}+m_{\pi}}{m_{D}}\right)^{2}\right)\left(1-\left(\frac{m_{K}-m_{\pi}}{m_{D}}\right)^{2}\right)\right]^{\frac{1}{2}} \\
& \times \frac{\left|V_{c s} F_{0}^{D K}\left(m_{\pi}^{2}\right)\right|^{2}}{\left|V_{c s} F_{0}^{D K}(0)\right|^{2}} \frac{a_{1}^{2}}{I^{D K}} \\
& \quad=0.225 \times \frac{a_{1}^{2}}{I^{D K}},
\end{aligned}
$$

where we used the fact $F_{0}^{D K}\left(m_{\pi}^{2}\right) \simeq F_{0}^{D K}(0)$ and the following experimental values [1 $\left.1 \overline{7}_{1}\right]: m_{D}=$ $m_{D^{0}}=1.8646 \pm 0.0005 \mathrm{GeV}, m_{K}=m_{K^{-}}=$ $493.677 \pm 0.013 \mathrm{MeV}, m_{\pi}=m_{\pi^{+}}=139.56995 \pm$ $0.00035 \mathrm{MeV}, f_{\pi}=f_{\pi^{+}}=131.74 \pm 0.15 \mathrm{MeV}$ and $V_{u d}=0.9753 \pm 0.0008$.

When we use the experimental results $\mathcal{B}\left(D^{0} \rightarrow\right.$ $\left.K^{-} \pi^{+}\right)=3.85 \pm 0.09 \%$ and $\mathcal{B}\left(D^{0} \rightarrow K^{-} e^{+} \nu\right)=$ $3.66 \pm 0.18 \%$, (造..$\overline{8})$ gives

$$
\begin{aligned}
& I^{D K}[\text { Expt. }]=0.213(1 \pm 0.054) a_{1}^{2} \\
= & 0.258(1 \pm 0.054)(1 \pm 0.091) \\
= & 0.258(1 \pm 0.106)=0.231 \sim 0.286,
\end{aligned}
$$

where we used the value of $a_{1}$ given in (5.4). On the other hand, when we calculate $I^{D K}$ directly from (5.7.) with $m_{F_{1}}=2.11 \mathrm{GeV}$ geven in Table 2 , we obtain the following results:

$$
\begin{aligned}
& I^{D K}\left[n_{F_{1}}=1\right]=0.195 \text { for } n_{F_{1}}=1, \\
& I^{D K}\left[n_{F_{1}}=2\right]=0.267 \text { for } n_{F_{1}}=2 .
\end{aligned}
$$

From $(5.9)$ and $\left(5 . \overline{1} 0^{i}\right)$, we find that the experimental results of $\mathcal{B}\left(D^{0} \rightarrow K^{-} \pi^{+}\right)$and $\mathcal{B}\left(D^{0} \rightarrow\right.$ $\left.K^{-} e^{+} \nu\right)$ together with the factorization assumption imply $n_{F_{1}}=2$.

In the same way as the above, for the $D$ to $\pi$ transition we get the formula

$$
\begin{aligned}
& \frac{\mathcal{B}\left(D^{0} \rightarrow \pi^{-} \pi^{+}\right)}{\mathcal{B}\left(D^{0} \rightarrow \pi^{-} e^{+} \nu\right)}=6 \pi^{2}\left|V_{u d}\right|^{2} \frac{f_{\pi}^{2}}{m_{D}^{2}}\left(1-\frac{m_{\pi}^{2}}{m_{D}^{2}}\right)^{2} \\
& \times\left[\left(1-\left(\frac{m_{\pi}+m_{\pi}}{m_{D}}\right)^{2}\right)\right]^{\frac{1}{2}} \frac{\left|V_{u d} F_{0}^{D K}\left(m_{\pi}^{2}\right)\right|^{2}}{\left|V_{u d} F_{0}^{D K}(0)\right|^{2}} \frac{a_{1}^{2}}{I^{D \pi}} \\
& =0.275 \times \frac{a_{1}^{2}}{I^{D \pi}},
\end{aligned}
$$

where we used the fact $F_{0}^{D \pi}\left(m_{\pi}^{2}\right) \simeq F_{0}^{D \pi}(0)$, and the dimensionless integral $I^{D \pi}$ is given by

$I^{D \pi}=\int_{0}^{\left(1-\frac{m_{\pi}}{m_{D}}\right)^{2}} d x \frac{\left(\left(1+\frac{m_{\pi}^{2}}{m_{D}^{2}}-x\right)^{2}-4 \frac{m_{\pi}^{2}}{m_{D}^{2}}\right)^{\frac{3}{2}}}{\left(1-\frac{m_{D}^{2}}{m_{F_{1}}} x\right)^{2 n_{F_{1}}}}$.

When we use the experimental results $\mathcal{B}\left(D^{0} \rightarrow\right.$ $\left.\pi^{-} \pi^{+}\right)=(1.53 \pm 0.09) \times 10^{-3}$ and $\mathcal{B}\left(D^{0} \rightarrow \pi^{-} e^{+} \nu\right)=$ $(3.7 \pm 0.6) \times 10^{-3},(\underline{5} .1 \overline{1})$ gives

$$
\begin{aligned}
& I^{D \pi}[\text { Expt. }]=0.665(1 \pm 0.173) a_{1}^{2} \\
= & 0.804(1 \pm 0.173)(1 \pm 0.091) \\
= & 0.804(1 \pm 0.195)=0.648 \sim 0.961,(5.13
\end{aligned}
$$

where we used again the value of $a_{1}$ given in (5).4. When we calculate $I^{D \pi}$ directly from (5.7) with $m_{F_{1}}=2.01 \mathrm{GeV}$ given in Table 2 , we obtain the following results:

$$
\begin{aligned}
& I^{D \pi}\left[n_{F_{1}}=1\right]=0.385 \text { for } n_{F_{1}}=1, \\
& I^{D \pi}\left[n_{F_{1}}=2\right]=0.783 \text { for } n_{F_{1}}=2 .
\end{aligned}
$$

The results in ( $(15 . \overline{1} \cdot \overline{4})$ show that the value of $I^{D \pi}$ is more sensitive to $n_{F_{1}}$ than that of $I^{D K}$. From $(\overline{1} \cdot \overline{1} \overline{3})$ and $(\bar{s} . \overline{1} \overline{4})$, we find that the experimental results of $\mathcal{B}\left(\bar{D}^{0} \rightarrow \pi^{-} \pi^{+}\right)$and $\mathcal{B}\left(D^{0} \rightarrow \pi^{-} e^{+} \nu\right)$ imply $n_{F_{1}}=2$. 
Table 8: The obtained branching fractions and their ratio for $\bar{B}^{0} \rightarrow D^{(*)+} l^{-} \bar{\nu}$

\begin{tabular}{|c|c|c|}
\hline$l$ & $\mathcal{B}\left(\bar{B}^{0} \rightarrow D^{+} l^{-} \bar{\nu}\right)$ & $\mathcal{B}\left(\bar{B}^{0} \rightarrow D^{*+} l^{-} \bar{\nu}\right)$ \\
& Ratio & Ratio \\
\hline$e$ & $\left(1.85_{+0.56}^{-0.47}\right) \times 10^{-2}$ & $\left(4.76_{+0.50}^{-0.47}\right) \times 10^{-2}$ \\
& 1 & 1 \\
$\mu$ & $\left(1.84_{+0.56}^{-0.47}\right) \times 10^{-2}$ & $\left(4.74_{+0.50}^{-0.46}\right) \times 10^{-2}$ \\
& $0.996_{-0.001}^{+0.001}$ & $0.996_{-0.000}^{+0.002}$ \\
$\tau$ & $\left(0.52_{+0.07}^{-0.07}\right) \times 10^{-2}$ & $\left(1.22_{+0.06}^{-0.06}\right) \times 10^{-2}$ \\
& $0.278_{-0.035}^{+0.049}$ & $0.256_{-0.013}^{+0.014}$ \\
\hline
\end{tabular}

\section{6. $\bar{B}^{0} \rightarrow D^{(*)+} l^{-} \bar{\nu}$}

We use the form factors in $(\underline{2} . \overline{8})$ with the following $\mathcal{F}_{D}(y)$ which are experimentally determined [1] 1 -

$$
\begin{gathered}
\mathcal{F}(y)=\mathcal{F}(1)\left[1-\rho^{2}(y-1)\right], \\
\left\{\begin{array}{cc}
\rho_{D}^{2}=0.59 \pm 0.25, & \left|V_{c b}\right| \mathcal{F}_{D}(1) \times 10^{2}=3.37 \\
& \text { for } \mathcal{F}_{D}(y) \\
\rho_{D^{*}}^{2}=0.84 \pm 0.15, & \left|V_{c b}\right| \mathcal{F}_{D^{*}}(1) \times 10^{2}=3.51 \\
\text { for } \mathcal{F}_{D^{*}}(y) .
\end{array}\right.
\end{gathered}
$$

By using the form factors in $\left(\underline{2}-\overline{8} \overline{8}_{1}^{-}\right)$with $\mathcal{F}_{D}(y)$ in $(6.1)$, we obtain from (12.2. $)$ the spectra of $\bar{B}^{0} \rightarrow$ $D^{+} l^{-} \bar{\nu}$ presented in Figure 3, where we find that the spectrum for muon drops down near $q^{2}=$ 0 . (The spectrum for electron also drops down near the very end of $q^{2}=0$.) We present the obtained branching fractions and their ratio in Table 8 , which gives $\mathcal{B}\left(\bar{B}^{0} \rightarrow D^{+} \tau^{-} \bar{\nu}\right) / \mathcal{B}\left(\bar{B}^{0} \rightarrow\right.$ $\left.D^{+} e^{-} \bar{\nu}\right)=0.278_{-0.035}^{+0.049}$ in good agreement with the ALEPH estimation $0.29 \pm 0.08$ 1. Since the HQET provides good informations about the heavy to heavy form factors, our results for the exclusive $B$ to $D$ semileptonic decays are very reliable.

By using the form factors in $(2.8)$ with $\mathcal{F}_{D^{*}}(y)$ in (6.11), we obtain from (12.7) the spectra of $\bar{B}^{0} \rightarrow$ $D^{*+} l^{-} \bar{\nu}$ presented in Figure 4. We present the obtained branching fractions and their ratio in Table 8 , where we find that $\mathcal{B}\left(\bar{B}^{0} \rightarrow D^{*+} \tau^{-} \bar{\nu}\right) /$ $\mathcal{B}\left(\bar{B}^{0} \rightarrow D^{*+} e^{-} \bar{\nu}\right)=0.256_{-0.013}^{+0.014}$ which is smaller than the ALEPH estimation $0.37 \pm 0.08$ [1] $\left.{ }^{1}\right]$.

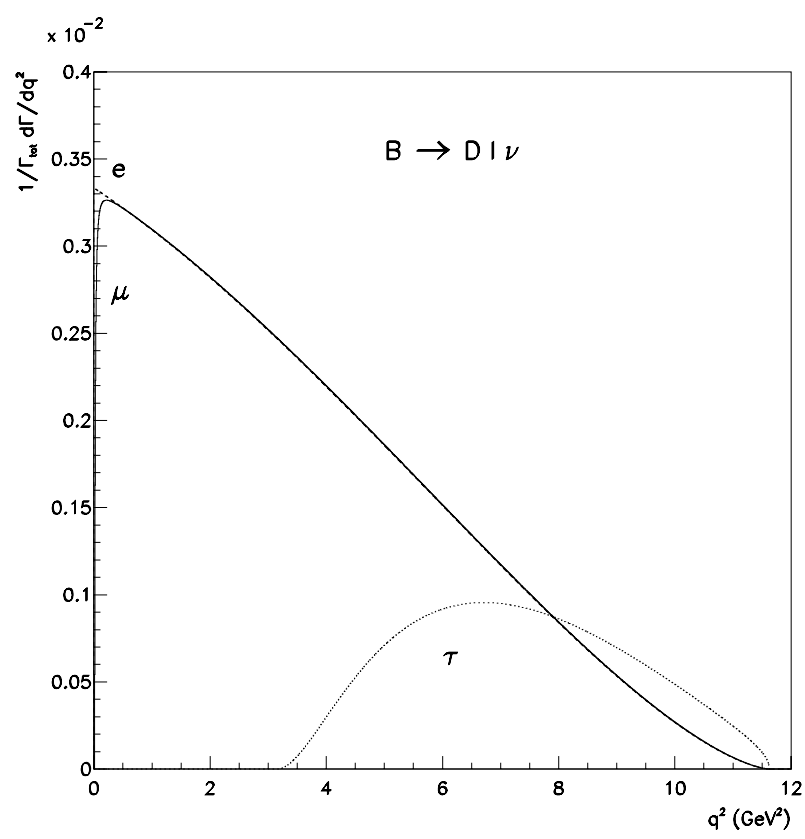

Figure 3: $\left(1 / \Gamma_{t o t}\right)\left(d \Gamma / d q^{2}\right)$ of $\bar{B}^{0} \rightarrow D^{+} l^{-} \bar{\nu}$ for $l=e, \mu$ and $\tau$.

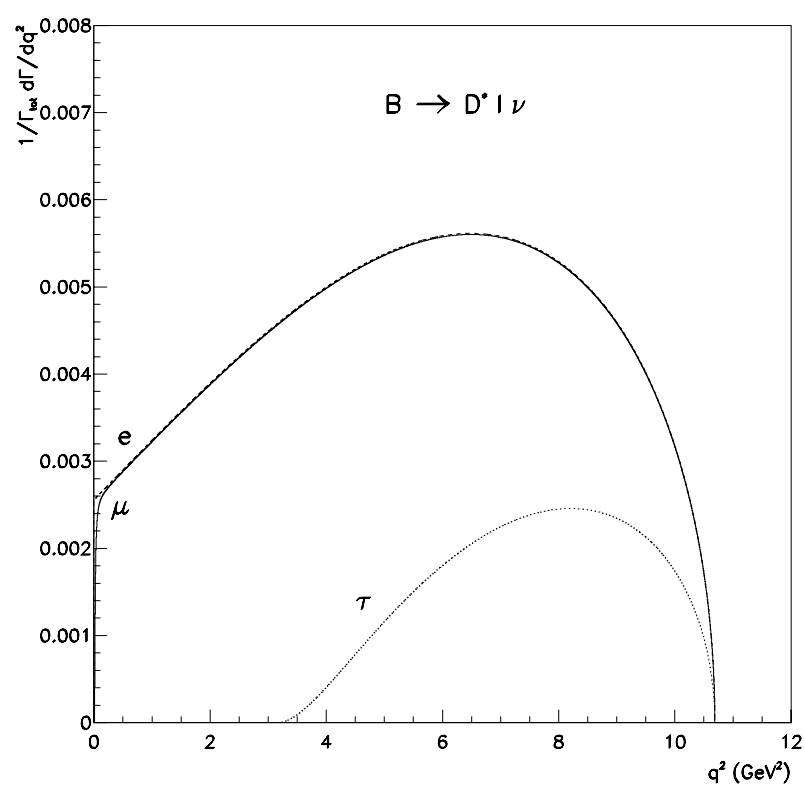

Figure 4: $\left(1 / \Gamma_{t o t}\right)\left(d \Gamma / d q^{2}\right)$ of $\bar{B}^{0} \rightarrow D^{*+} l^{-} \bar{\nu}$ for $l=e, \mu$ and $\tau$.

\section{Conclusion}

We studied the $D$ meson exclusive semileptonic decays and investigated their sensitivity to form factor models. The results show that the decays to a pseudoscalar meson and a lepton pair are sensitive to the property of the form factor 
$F_{1}\left(q^{2}\right)$, and those to a vector meson and a lepton pair are sensitive to the form factor $A_{1}\left(q^{2}\right)$. Experimentally $F_{1}^{D K}(0)=0.75 \pm 0.02 \pm 0.02$ has been extracted from the branching fraction $\mathcal{B}\left(D^{0} \rightarrow\right.$ $\left.K^{-} e^{+} \nu\right)=(3.68 \pm 0.21) \times 10^{-2}$ by assuming that $F_{1}^{D K}\left(q^{2}\right)$ is of simple pole type $\left(n_{F_{1}}=1\right)$ [1] $3_{1}^{\prime}$. However, at present the $q^{2}$ dependence of $F_{1}\left(q^{2}\right)$ is not settled experimentally or theoretically. We analyzed the ratios of the branching fractions $\mathcal{B}\left(D^{0} \rightarrow K^{-}\left(\right.\right.$or $\left.\left.\pi^{-}\right) \pi^{+}\right)$and $\mathcal{B}\left(D^{0} \rightarrow\right.$ $K^{-}\left(\right.$or $\left.\left.\pi^{-}\right) e^{+} \nu\right)$ using the factorization assumption, and found that it is implied that the form factor $F_{1}\left(q^{2}\right)$ of the $D$ to $K$ and that of the $D$ to $\pi$ transitions are of dipole type $\left(n_{F_{1}}=2\right)$.

If we extract $F_{1}^{D K}(0)$ from $\mathcal{B}\left(D^{0} \rightarrow K^{-} e^{+} \nu\right)=$ $(3.68 \pm 0.21) \times 10^{-2}$ by assuming $n_{F_{1}}=2$ instead of $n_{F_{1}}=1$, we get $F_{1}^{D K}(0)=0.64 \pm 0.03$ which is substantially different from the above value 0.75 . The experimental extraction of the reliable value of $F_{1}^{D K}(0)$ is important for the determinations of the fundamental parameters, and also since the experimentally extracted value of $F_{1}^{D K}(0)$ is compared with its values obtained by different theoretical calculations such as quark models, lattice QCD, and QCD sum rules [1] 114]. The extraction of the value of $\left|V_{u b} F_{1}^{B \pi}(0)\right|$ from the measured semileptonic branching fraction $\mathcal{B}\left(B^{0} \rightarrow \pi^{-} l^{+} \nu\right)$ is also dependent on the model for $F_{1}^{B \pi}\left(q^{2}\right)$ emplyed, and this dependence is even bigger than the case of the above $F_{1}^{D K}(0)$ extraction.

\section{Acknowledgements}

DSH is grateful to Helen Quinn for helpful suggestions. He is also thankful to Jeffrey Appel, Harry Cheung, Karl Ecklund, Will Johns, Peter Kim, Noel Stanton, and Sheldon Stone for interesting and useful discussions on the experimental aspects of this work. This work was supported by Non-Directed-Research-Fund, Korea Research Foundation 1997, by the Basic Science Research Institute Program, Ministry of Education, Project No. BSRI-97-2414, by the Korea Science and Engineering Foundation, Grant 9850200-002-2, and by the Research Fund of Kangnung National University 1997.

\section{References}

[1] N. Cabibbo, Phys. Rev. Lett. 10, 531 (1963); M. Kobayashi and T. Maskawa, Prog. Theor. Phys. 49, 652 (1973).

[2] J.G. Körner and G.A. Schuler, Phys. Lett. B 231, 306 (1989); Z. Phys. C 46, 93 (1990).

[3] A. Khodjamirian, R. Rückl and C.W. Winhart, Phys. Rev. D 58, 054013 (1998).

[4] D.S. Hwang and D.-W. Kim, hep-ph/9806242 (1998).

[5] F.J. Gilman and R.L. Singleton, Jr., Phys. Rev. D 41, 142 (1990).

[6] UKQCD Collaboration, K.C. Bowler et al., Phys. Rev. D 51, 4905 (1995).

[7] N. Isgur and M.B. Wise, Phys. Lett. B 232, 113 (1989); 237, 527 (1990).

[8] M. Wirbel, B. Stech and M. Bauer, Z. Phys. C 29, 637 (1985); 34, 103 (1987); M. Bauer and M. Wirbel, Z. Phys. C 42, 671 (1989).

[9] D.S. Hwang and B.-H. Lee, Eur. Phys. J. C 6, 663 (1999).

[10] UKQCD Collaboration, D.R. Burford et al., Nucl. Phys. B 447, 425 (1995); L.D. Debbio, Phys. Lett. B 416, 392 (1998).

[11] E687 Collaboration, P.L. Frabetti et al., Phys. Lett. B 364, 127 (1995).

[12] H.W.K. Cheung and W. Johns, Private Communications.

[13] R.J. Morrison and J.D. Richman, Note on semileptonic decays of $D$ and $B$ mesons, Part I (in Particle Data Group 1994), Phys. Rev. D 50, 1565 (1994).

[14] A. Ryd, Review of Form Factor Measurements, in Seventh International Symposium on heavy Flavor Physics, Santa Barbara, July, 1997.

[15] E687 Collaboration, P.L. Frabetti et al., Phys. Lett. B 382, 312 (1996).

[16] M. Neubert and B. Stech, hep-ph/9705292, to appear in second edition of Heavy Flavours, ed. by A.J. Buras and M. Lindner, World Scientific, Singapore.

[17] Review of Particle Physics 1998, Eur. Phys. J. C 3, 1 (1998).

[18] CLEO Collaboration, B. Barish et al., Phys. Rev. D 51, 1014 (1995); M. Athanas et al., Phys. Rev. Lett. 79, 2208 (1997).

[19] ALEPH Collaboration, D. Buskulic et al., Phys. Lett. B 343, 444 (1995); 395, 373 (1997). 